/04/ 


\section{EDUCACIÓN CONTINUA, GESTOR DEL APRENDIZAJE Y CONOCIMIENTO EN LA EDUCACIÓN SUPERIOR}

\section{CONTINUING EDUCATION, MANAGER OF LEARNING AND KNOWLEDGE IN HIGHER EDUCATION}

Carlos Fernando Meléndez Tamayo

Doctor en Educación por la Universidad Complutense de Madrid, Magister en Gerencia de la Educación Abierta, Especialista en Proyectos de Investigación Científica y Tecnológica, Ingeniero en Sistemas Informáticos, Profesor - Investigador, Director del Departamento de Educación a Distancia y Virtual de la Universidad Técnica de Ambato (UTA). (Ecuador) E-mail: cmelendez77@uta.edu.ec ORCID: https://orcid.org/0000-0002-7990-4859

Luis Danilo Flores Rivera

Magister en Arquitectura de la Información, Magister en Educación a Distancia, Ingeniero en Electrónica e Instrumentación, Ingeniero de Mantenimiento, Ingeniero Comercial, Personal de Apoyo Académico - Técnico Docente de la Universidad Regional Amazónica IKIAM. (Ecuador) E-mail: 1daniflores77@gmail.com ORCID: https://orcid.org/0000-0003-1301-6880

Recepción: 15/09/2018. Aceptación: 12/12/2018. Publicación: 28/12/2018

Citación sugerida:

Meléndez Tamayo C. F. y Flores Rivera, L. D. (2018). Educación Continua, Gestor del Aprendizaje y conocimiento en la Educación Superior. 3C TIC. Cuadernos de desarrollo aplicados a las TIC, 7(4), pp.76-97. doi:http://dx.doi.org/10.17993/3ctic.2018.62.76-97 


\section{RESUMEN}

La presente investigación tiene como propósito conocer los escenarios, metodologías, modalidades y medios tecnológicos en los que la educación superior se desarrolla. La Universidad como una entidad humanística, formadora y creadora de la investigación, la cultura, y la ciencia son el pilar fundamental en la gestión de aprendizaje. Es así que la misma ha generado y adoptado sistemas inteligentes de aprendizaje que han evolucionado de acuerdo a las necesidades y competencias requeridas por la sociedad, siendo la educación continua uno de los procesos que la Universidad ha vinculado al servicio de las personas en general, estudiantes, docentes y profesionales. Este medio ha permitido ser aporte y soporte de la gestión del conocimiento, con talento humano y estructura necesaria para su trabajo. La investigación analizó una muestra de 75 participantes de los cursos de educación continua de la Dirección de Educación a Distancia y Virtual (DEaDV) de la Universidad Técnica de Ambato (UTA).

El procedimiento metodológico que respaldó la investigación fue análisis cuali-cuantitativo, seguida de la investigación bibliográfica-documental, que profundizó diferentes enfoques, teorías, conceptualizaciones y criterios de diversos autores. Posteriormente, se aplicó la investigación de campo a los dos cursos de educación continua, por medio de un cuestionario estructurado, el cual había sido validado previamente en tres fases, obteniéndose resultados para el análisis descriptivo, que permitieron plantear conclusiones y recomendaciones de la investigación.

El proceso permitió evidenciar las nuevas estructuras para gestionar la información y conocimiento en la educación superior, así como exponer resultados de diferentes ámbitos. Entre los más destacados están la evaluación de la estructura y la organización metodológica curricular con una aceptación del $87 \%$, la pertinencia de utilizar productos acreditables (actividades, tareas, informes, trabajos finales) con un $73 \%$ de consideración y la preferencia de la modalidad híbrida o b-learning con el $84 \%$.

\section{ABSTRACT}

The purpose of this research is to know the scenarios, methodologies, modalities and technological means in which higher education develops. The University as a humanistic entity that forms and creates research, culture and science is the main base in the management of learning. Therefore, the University has generated and adopted intelligent learning systems that have evolved according to the needs and competencies required by society, being continuous education one of the processes that the University has linked to the service of people in general, students, teachers and professionals. This means has allowed 
to be contribution and support of the knowledge management, with human talent and necessary structure for its work. The research analyzed a sample of 75 participants of the continuing education courses of the Directorate of Distance and Virtual Education (DEaDV) of the Technical University of Ambato (UTA).

The methodological procedure that supported the research was qualitative-quantitative analysis, followed by bibliographicdocumentary research that deepened different approaches, theories, conceptualizations and criteria of different authors. Subsequently, field research was applied to the two continuing education courses, by means of a structured questionnaire, which prior to the application was validated in three phases, obtaining results for the descriptive analysis, which allowed to raise conclusions and recommendations of the research.

The process made it possible to demonstrate the new structures for managing information and knowledge in higher education, as well as expose results from different areas, among the most outstanding the evaluation of the structure and the curricular methodological organization with an acceptance of $87 \%$, the relevance of using creditable products (activities, tasks, reports, final works) with a $73 \%$ consideration and the preference of the hybrid or b-learning modality with 84\%.

\section{PALABRAS CLAVE}

Educación 4.0, Educación continua, Educación superior, Gestión del conocimiento, Sistemas inteligentes.

\section{KEYWORDS}

Education 4.0, Continuing education, Higher education, Knowledge management, Intelligent systems.

\section{INTRODUCCIÓN}

La Educación Continua se ha constituido en el medio dinámico en que la actividad docente universitaria se vincula con programas de formación y capacitación al educando (profesionales, estudiantes, público en general) que desee o requiera profundizar conocimientos, habilidades, actitudes y destrezas que fortalecen su campo de acción, o quiera ampliar conocimientos a áreas complementarias, emplear nuevos procedimientos y/o tecnologías con el fin de lograr un mejor desempeño, posición laboral y un desarrollo personal (Red Universitaria de Educación Continua, 2017).

Un factor significativo constituye la innovación tecnológica que ha planteado nuevos escenarios para la educación, donde están inmersas las tecnologías de la información y comunicación (TIC), la robótica, la inteligencia artificial (IA), sistemas expertos, realidad virtual (RV), realidad aumentada 
(RA), la industria 4.0, Internet de las Cosas (Industrial Internet of Things [IoT]), minería de datos (data mining), inteligencia de datos (big data) y las relacionadas con la inteligencia emocional, aprendizaje participativo (coaching), mejoramiento en el nivel de atención (mindfullnes). Estas tecnologías y conceptos modernos deben tener conciencia colectiva y socioformación como punto de unión y equilibrio a través de una colaboración continua (Fierro Santillán y Alfredo Díaz, 2017).

Del contexto indicado, las TIC tienen alta importancia puesto que han influenciado en la gestión, colaboración de entornos virtuales y metodología utilizada en el proceso enseñanza aprendizaje (PEA) (López Tórrez y López Herández, 2015). Por elllo, se están integrando la tendencia de la industria 4.0, que habla del conocimiento que es necesario pero no es suficiente, con relación al razonamiento abstracto y el conocimiento que requieren estar vinculados con el mundo real a través de proyectos y aplicaciones reales. Esto conlleva a que la Universidad, instituciones de educación superior y centros educativos deban internalizar una metodología de aprendizaje en la que alumnos y profesores colaboren para diseñar proyectos para la vida real que se desarrollan en equipo durante semanas o incluso meses (Ranz, 2016).

Esta perspectiva evidencia que la tecnología es esencial en el aprendizaje universitario, puesto que fomenta la cultura investigativa, la innovación de aprendizajes y la retroalimentación de conocimientos (Flores Rivera y Meléndez Tamayo, 2017), pero su cumplimiento se dará siempre y cuando el entorno organizacional sea inteligente, y con esto se quiere decir conocimiento $y$ aprendizaje del proceso, adaptación a los cambios culturales (Garbanzo-Vargas, 2016) y evolución del rol docente (capital humano), factor clave para alcanzar la calidad en la innovación digital. Esta transformación se dará con la enseñanza superior reorientando la formación y aumentando el sector de producción TIC (producción de software y servicios digitales), acompañada de programas de educación continua que sean mecanismos de inclusión de sectores sociales desfavorecidos, que abarquen TIC con todos los programas y carreras específicas en esta disciplina (Bedoya Rodríguez, et al., 2016). Estos factores deben complementarse con una gestión eficiente y efectiva por parte de la institución educativa, y asimismo ejercer una responsabilidad social que contribuya al desarrollo en un contexto global y la praxis de mejoramiento continuo que permita alcanzar estándares de calidad académica. 


\section{REVOLUCIÓN TECNOLÓGICA}

Los avances e innovaciones tecnológicas desarrollados con la digitalización han revolucionado los ámbitos social, económico, cultural y educativo. Esto se está palpando y será con mayor intensidad en el futuro. El Internet y las tecnologías de vanguardia están en un constante crecimiento, con lo cual será fácil vincular fábricas inteligentes, industria 4.0, IoT, la robótica industrial,... entre otras tendencias que la sociedad va priorizando. Con lo dicho es vital que la educación innove y educar para innovar, en otras palabras, replantear las formas de aprender, enseñar, construir y compartir la información y conocimientos (Restrepo, 2016).

En la Figura 1, se ilustra la evolución de las etapas tecnológicas versus el grado de cambios e impactos en la modernidad industrial.

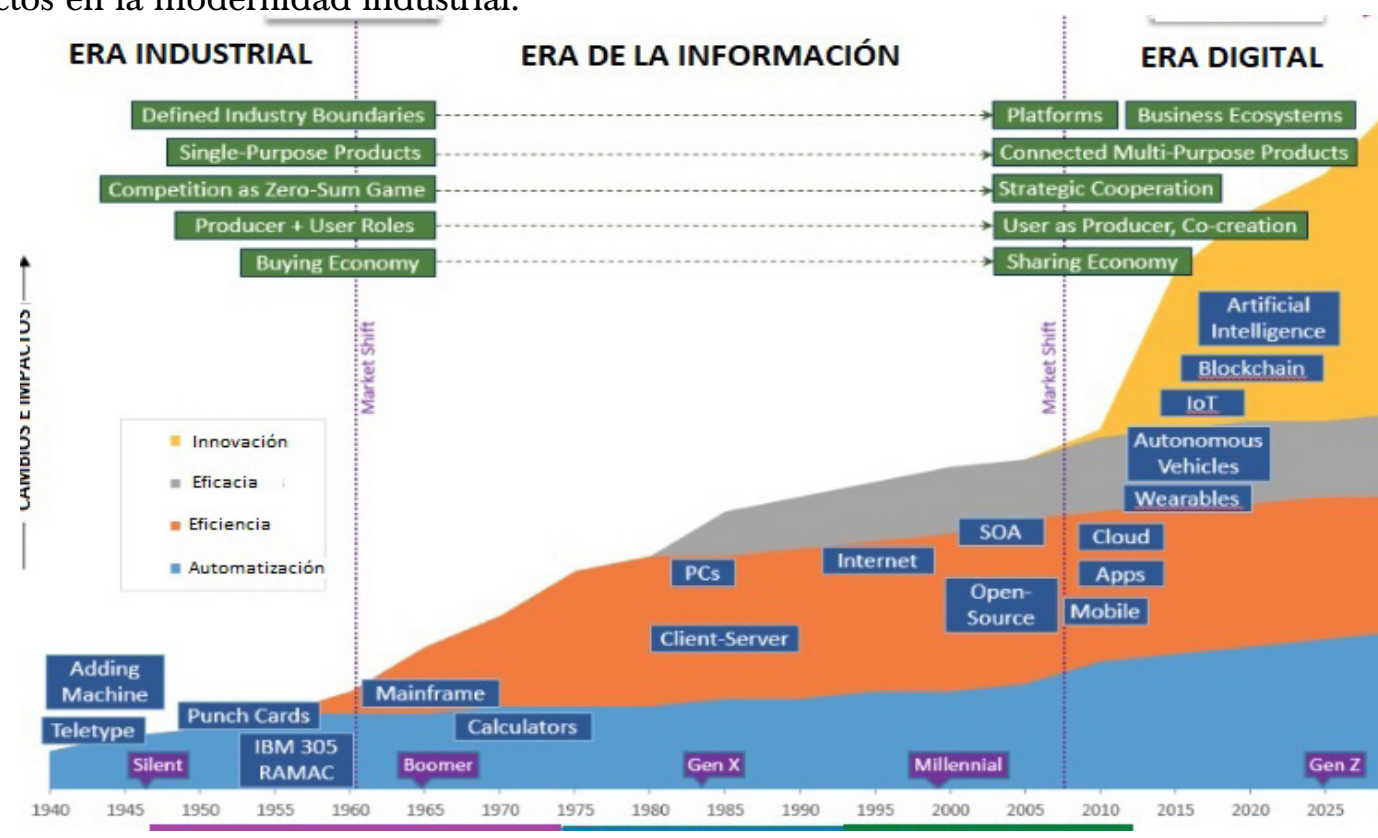

Figura 1. Evolución de las etapas tecnológicas y el grado de cambios e impactos en la modernidad industrial.

Fuente: Majesco, (2016). 
De la Figura 1 se puede extraer que en la era industrial está presente la automatización y que al final de la misma se ponen en marcha criterios de eficiencia. En la era de la información, la automatización tiene un leve incremento, se destaca mayor incidencia de la eficiencia en los procesos productivos y surge el concepto de eficacia como criterio de optimizar recursos y la productividad. La era digital tiene incremento en el índice de automatización, eficiencia y eficacia en los procesos, concentrándose mayores esfuerzos en la innovación de productos y servicios. En esta etapa sobresalen las IoT, la inteligencia artificial y las cadenas de bloques (base de datos que enlaza la recuperación de información y la verificación que no se ha cambiado [blockchain]) (criptonoticias, 2015).

Otro aspecto que registrar por los cambios industriales es las características del mercado en la modernidad digital:

- De límites definidos por la industria a escenarios donde están presentes plataformas y ecosistemas de negocios.

- De la competencia de juego de suma cero (la ganancia o pérdida de un participante se equilibra) a un usuario como productor participe de la creación.

- De economía de compra a economía colaborativa.

Además, la modernidad digital asociada a la revolución en la industria 4.0, se caracteriza por tres atributos esenciales: más flexibilidad en procesos de producción a medida de cada cliente individual, más velocidad para reducir a un tiempo óptimo y colocar el producto en el mercado y mayor eficiencia-eficacia por técnicas del análisis de datos que permite la digitalización y el IoT (Ranz, 2016). Estos eventos paralelamente se relacionan con la educación 4.0.

\subsection{EDUCACIÓN 4.0}

La educación 4.0, que tiene tres características fundamentales:

- Aprendizaje flexible en función de las necesidades e intereses de cada estudiante.

- Aprendizaje al propio ritmo y a la velocidad de cada estudiante con independencia de su edad, curso y afinidad. 
- Aprendizaje digital con retroalimentación (feedback) constante a partir del análisis de los datos derivados del progreso del propio aprendizaje (Learning Analytics).

Lo primordial en la educación 4.0 es el aprendizaje con factores de flexibilidad, ritmo propio y análisis de datos. A la par va la transformación digital, medio en que la educación 4.0, marca una tendencia tecnológica, velocidad en sus procesos y nuevos desafíos académicos que son imparables con gran impacto e irreversibles. En este sentido, la tecnología es un catalizador clave del proceso de transformación digital de la educación.

\section{Retos de la educación 4.0}

La complejidad en los retos de la educación 4.0, es la gestión a realizarse por los líderes educativos y son las siguientes:

- El cambio de las prácticas sociales y cultura en las instituciones educativas, universidades y administraciones públicas.

- La formación del profesorado y de equipos directivos.

- El estímulo, atracción y desarrollo del talento de los profesores a la educación 4.0. (Ranz, 2016).

\subsection{SISTEMAS INTELIGENTES EN LA GESTIÓN DE EDUCACIÓN MASIVA}

El desarrollo de sistemas informáticos ha generado una transformación en la educación, dando paso a ambientes, entornos, escenarios y/o plataformas de aprendizaje, sobresaliendo los siguientes:

- Cursos en línea basados en la gratuidad y la apertura de contenidos a todo tipo de usuarios (Massive Online Open Courses [MOOCs]).

Los MOOCs, son los más novedosos de hoy en día, su idea surgió en 2001 con el concepto Aprendizaje Social (Social Learning), que es la posibilidad de aprender a través de la interacción con otras personas. Los MOOCs alcanzan su popularidad en 2011 gracias al curso Introduction to Artificial Intelligence ofrecido por Sebastian Thrun y Peter Norvig en la Universidad de Stanford a través de la compañía startup llamada Know Labs (actualmente Udacity), donde se matricularon más de 160.000 personas. Esto fue el precedente a diferentes plataformas como Coursera o edX (Vivar Zurita, Vinader Segura y Abuín Vences, 2015). 
El escenario y enfoque de los cursos es la masividad, donde las personas pueden tener acceso a un nivel de acreditación de las Universidades reconocidas en el mundo entero. Esto es factible con la incorporación de las TIC, Inteligencia Artificial, Sistemas de Redes Neuronales Artificiales y otras Tecnologías Inteligentes que permitan acreditar los procesos curriculares de aprendizaje y evaluación (Diaz, 2014).

Lo renovado de los MOOC es su clasificación, sin existir unanimidad de estudios e importancia pueden ser:

- cMOOC, cursos gratuitos y verdaderamente accesibles que buscan el aprendizaje basándose fundamentalmente en la teoría conectivista, que es interacción con otros estudiantes (a través de blogs, feeds RSS) y son precisamente estas relaciones el valor agregado, pasando la certificación segundo plano y facilitando la creatividad y el trabajo colaborativo en red.

- xMOOC, no conectivistas, se centran contenidos ofrecidos únicamente a través de la página web y permite gestionar gran número de estudiantes inscritos. En este caso, la colaboración es más rígida y la acreditación del curso se mide a través de la evaluación de pruebas o trabajos (Vivar Zurita, Vinader Segura y Abuín Vences, 2015).

Con lo señalado, es vital que los MOOCs evolucionen con una nueva visión del aprendizaje, fomentando la individualización y gestión de tecnologías digitales e inteligentes para obtener logros personales, como una alternativa a los cursos masivos tradicionales (Zapata-Ros, 2014).

\section{Inteligencia de datos (big data)}

La inteligencia de datos es un factor importante en la educación digital, permite mejor control y gestión de aprendizajes de los estudiantes; su mayor valor está en el tiempo real en que se pueden ejecutar actividades como captura, almacenamiento y procesamiento de datos. El docente puede monitorear, identificar y reprogramar los procesos que desarrolla el educando, con lo que se optimiza actividades para la comprensión en las distintas áreas en la que se prepara.

El big data ayuda al docente a crear una ruta personalizada para todo el proceso educativo. Esto permite al estudiante la mejor versión de sí mismo que es la meta de la educación. En este nuevo escenario, el docente pasa a hacer un coach del estudiante, buscando descubrir debilidades y fortalezas con el fin de trabajar, motivar y superar las dificultades presentadas e impulsar sus puntos 
fuertes. Este giro radical con un feedback en tiempo real promueve la eficiencia y dinamismo académico en la que están inmersos estudiantes, docentes y editores de contenidos.

La realidad del big bata será fortalecida con las entidades inmersas (gobierno, universidad, instituciones de educación y empresas tecnológicas) en el proceso educativo y que se alineen y emprendan un proyecto de transformación definitivo. El logro de estas acciones serán una sociedad mejor preparada, más creativa, menores tasas de desempleo, por tanto más competitiva (García Barbosa, García Esteban, de Rojas, López López y Nuñez, 2016). La Figura 2 describe el funcionamiento del big data.

Figura 2. Big data ¿Cómo funciona este proceso?

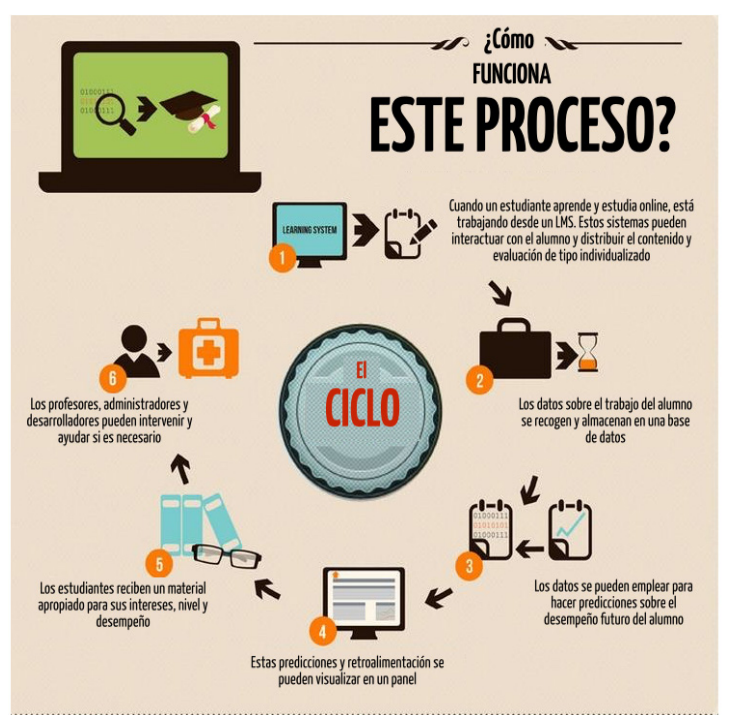

Fuente: The Flipped Classroom , (2014) 


\subsection{TENDENCIAS Y DESAFÍOS}

La educación superior en una investigación realizada por la New Media Consortium (NMC) y su proyecto horizonte (Horizon Project) presenta aspectos clave a ser considerados por las Instituciones, Autoridades de educación, Líderes de educación, Educadores y todo miembro partícipe de la academia. Dentro de las perspectivas están las tendencias, los desafíos significativos y los desarrollos tecnológicos, que tendrán un impacto global y serán de gran importancia en los próximos cinco años (Johnson, 2016). La Figura 3, relaciona las tendencias, desafíos y desarrollos tecnológicos.

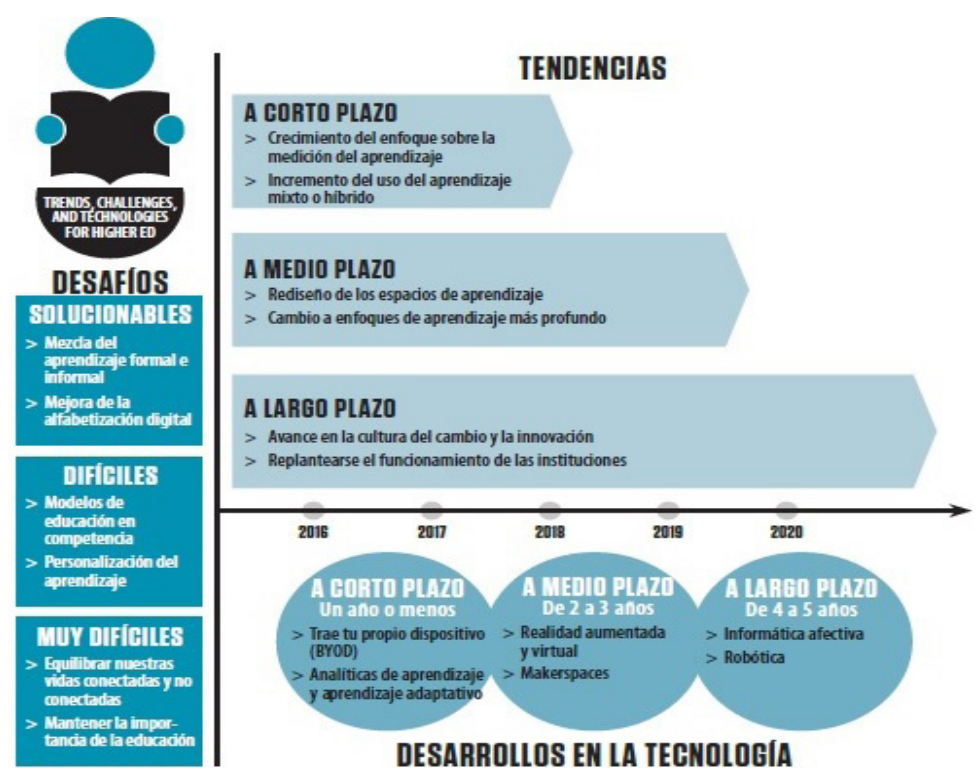

Figura 3. Tendencias, desafíos y desarrollos tecnológicos en la educación superior.

Fuente: Johnson, (2016).

El modelo NMC Horizon Project (2016) usa tres enfoques para las tendencias y desafíos: política (leyes formales, las regulaciones, las reglas y las directrices que gobiernan universidades y unidades educativas), liderazgo (visión de futuro de los expertos, basada en la investigación y la profunda consideración) y práctica (nuevas ideas y pedagogías, en universidades y entornos relacionados) (Johnson, 2016). 


\section{Desafíos significativos que impiden la adopción de tecnologías en la enseñanza superior}

\section{Desafíos solucionables: aquellos que entendemos y sabemos cómo solucionar}

\section{Mezcla del aprendizaje formal e informal}

Internet ha puesto todo al alcance de nuestra mano, promoviendo el interés por las clases de aprendizaje auto dirigidas basadas en la curiosidad que surgen comúnmente de museos, centros científicos y redes de aprendizaje personales. Estas y otras formas menos formales de aprendizaje recaen bajo el aprendizaje informal y sirven para mejorar la participación de estudiantes, y los anima a seguir sus propias vías de aprendizaje e intereses.

\section{Mejora de la alfabetización digital}

La proliferación de Internet, dispositivos móviles y otras tecnologías ahora omnipresentes en la educación, la visión tradicional de la alfabetización de leer y escribir se ha expandido en la comprensión de las herramientas digitales y la información. Por tanto, las instituciones de educación deben fortalecer sus competencias en problemas de alfabetización, objetivos curriculares y programas de desarrollo docente. La aceptación de la alfabetización digital está impidiendo a muchas escuelas y universidades formular políticas y programas que aborden este desafío de forma adecuada. El debate de la alfabetización digital como competencia de una serie de herramientas digitales con diferentes objetivos educativos, o como un indicador de la capacidad de evaluar críticamente los recursos disponibles en la web.

\section{Desafíos difíciles: aquellos que entendemos pero cuyas soluciones son imprecisas}

\section{Modelos de educación en competencia}

Los nuevos modelos de educación se contraponen a los modelos tradicionales de educación superior, donde los estudiantes reciben clases impartidas por docentes, asistiendo al campus a cambio de créditos académicos. Las instituciones buscan maneras de proporcionar servicios de aprendizaje de alta calidad y más oportunidades a menor costo. Las MOOCs, la educación basada en competencias, 
la codificación de los bootcamps (campos de entrenamiento) de programación, y la desagregación general de productos y servicios afectan a los sistemas de crédito-hora actuales y a los programas de grado. Las tendencias de sistemas de aprendizaje necesitan de líderes de educación que evalúen con veracidad los modelos y determinen la mejor manera de apoyar la colaboración, interacción y evaluación a gran escala.

\section{Personalización del aprendizaje}

La personalización del aprendizaje se relaciona con programas educativos, experiencias, métodos de enseñanza y estrategias de apoyo académico que abordan necesidades de aprendizaje, intereses, aspiraciones, o antecedentes culturales específicos de estudiantes individuales. Al aprendizaje personalizado, le falta el apoyo tecnológico y prácticas modernas, especialmente a gran escala. El creciente interés en la personalización de la enseñanza busca satisfacer necesidades particulares de los estudiantes y promueve el desarrollo tecnológico.

\section{Desafíos muy difíciles: aquellos que son difíciles incluso de definir y mucho más de solucionar}

\section{Equilibrar nuestras vidas conectadas y no conectadas}

Las instituciones de educación superior deben equilibrar el uso de las tecnologías con otras actividades necesarias para el desarrollo de los estudiantes. Por tanto, universidades e instituciones de educación superior tienen que fomentar un uso consiente de las herramientas digitales y los efectos de las mismas. La educación al estar armonizando con las tendencias tecnológicas, los instructores deben facilitar oportunidades donde los estudiantes sientan, digieran, reflexionen y lleven a cabo experiencias sensoriales cruciales para el desarrollo del carácter y la integridad.

\subsection{OBJETIVOS}

Los objetivos propuestos para el estudio:

- Conocer los escenarios, metodologías, modalidades y medios tecnológicos en los que la educación superior está desarrollando.

- Establecer nuevas estructuras que gestionen información y conocimientos a la educación superior. 


\section{METODOLOGÍA}

El análisis cuali-cuantitativo se utilizó para potenciar el enfoque de la investigación, como lo indica Pérez Juste (1994), la investigación educativa contempla la modalidad de investigación destinada a evaluar los programas educativos en condiciones de rigor de cara a la mejora de las personas a las que se aplica. Sus transcendentales características son la practicidad y la especificidad a realidades concretas, diversidad en los diseños de investigación desde el contexto cuantitativo y cualitativo.

Se utilizó la investigación bibliográfica-documental para ampliar, comparar, conocer, profundizar diferentes enfoques, teorías, conceptualizaciones y criterios de diversos autores sobre un tema determinado. Los medios que apoyaron esta investigación fueron revistas indexadas, blogs de expertos, libros, e-libros entre otros medios digitales.

Posteriormente, se aplicó investigación de campo extrayendo informaciones de los participantes de los cursos de educación continua dos paralelos dando una totalidad de 75 participantes; este contacto en forma directa permitió obtener impresiones de la realidad de los participantes, así como adquirir información vinculada a los objetivos del proyecto.

El instrumento aplicado para medir las impresiones de los participantes fue la encuesta con un cuestionario estructurado. Este se aplicó en el sitio en donde los participantes encuadran las actividades del curso. Una vez aplicado el instrumento, se procedió con la investigación descriptiva, con la cual se obtuvo resultados que permiten plantear conclusiones y recomendaciones de la investigación realizada.

Es importante mencionar que se realizó un proceso de validación del cuestionario, el cual fue desarrollado en tres fases:

1. Validación del cuestionario por parte de un experto, el mismo que permitió mejorar la estructura, redacción de las preguntas y relevancia para el consecuente procesamiento de la información.

2. Se rediseñó el cuestionario con los ítems, en base a las observaciones y sugerencias del experto.

3. Se aplicó una prueba piloto, en la que se pudo detectar dificultades por parte de los encuestados 
al momento de contestar las preguntas; ya sea por emplear un lenguaje extremadamente técnico o complejo. El proceso se realizó aleatoriamente en dos cursos de educación continua con un total de 75 participantes, en donde se impartía el curso de Docencia Universitaria. Los cursos son desarrollados por la Dirección de Educación a Distancia y Virtual (DEaDV) de la Universidad Técnica de Ambato (UTA) universidad pública del Ecuador.

\section{Modo de aplicación del instrumento}

Este proceso permitió obtener un instrumento de calidad, que fue clave para recolectar información confiable y relevante de los procesos que maneja los cursos de educación continua de la UTA.

Una vez validado el instrumento, se procedió con los siguientes pasos:

1. El instrumento "encuesta" fue enviado por vía electrónica mediante la notificación al correo electrónico del participante, mismo que fue registrado el momento de la inscripción, para lo cual se estableció un cronograma de aplicación del instrumento.

2. Se aplicó la encuesta a todos los participantes que en número fueron 600 de los cursos de educación continua de la DEaDV de la UTA.

3. Se cotejaron y registraron las respuestas de la encuesta aplicada al proceso.

4. Se tabularon los resultados obtenidos del proceso, dando paso al análisis y discusión de los mismos.

\section{RESULTADOS Y DISCUSIÓN}

Se evaluó la estructura metodológica planteada en la formación identificando que los elementos de mayor significación son:

a) Los productos acreditables por unidades.

b) Evaluación colaborativa obligatoria mediante la videoconferencia.

El $87 \%$ de los encuestados (Figura 4), manifestaron la conformidad con la metodología utilizada, entendiéndose como metodología la organización curricular del curso que contó con tres zonas: 
- Zona de información.

- Zona académica.

- Zona de cierre.

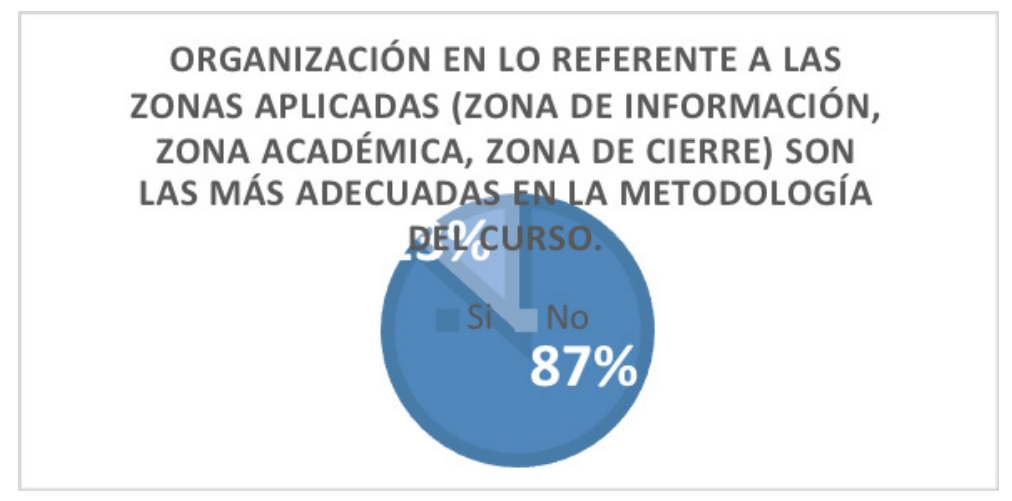

Figura 4. Organización en la Metodología del curso.

Fuente: sistema informático DEaDV-UTA (2017).

Tabla 1. Resultados organización en la Metodología del curso.

La organización en lo referente a las zonas aplicadas (zona de información, zona académica, zona de cierre) son las más adecuadas en la metodología del curso.

\begin{tabular}{cc}
\hline Sí & 65 \\
No & 10 \\
\hline
\end{tabular}

Nota: Tomada del Sistema informático DEaDV-UTA - Encuesta (2017).

La zona académica fue considerada la más relevante, ya que el 73\% de los encuestados encontraron la pertinencia al utilizar elementos como productos acreditables en actividades, tarea, informe, trabajo final evaluado, entre otros. Es importante mencionar que, existió una alta participación e interacción en foros (Figura 5), con retroalimentación continua la misma que reforzó el aprendizaje. 


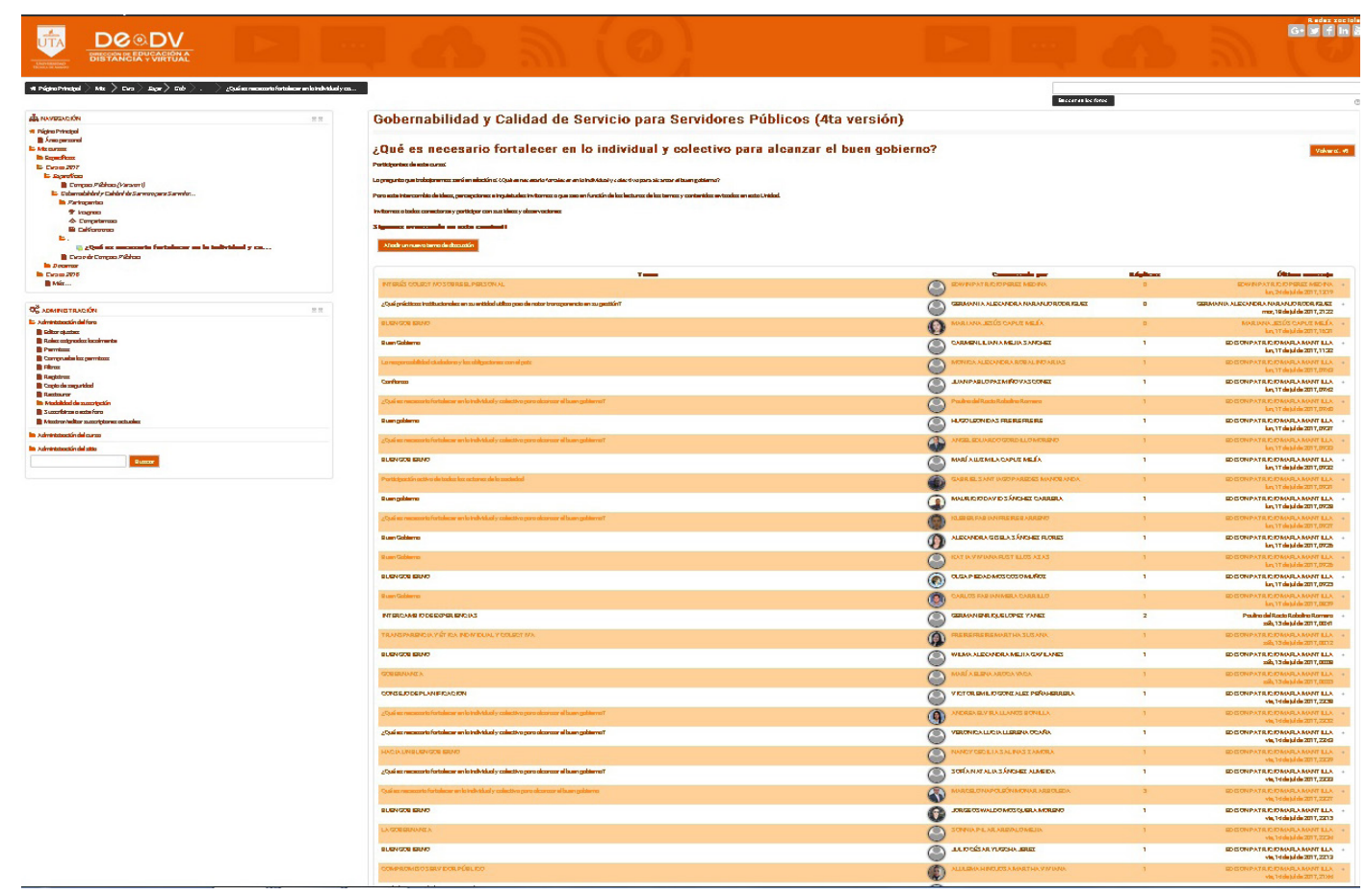

Figura 5. Interacción de los usuarios en los foros de los cursos de educación continua.

Fuente: Plataforma educativa DEaDV-UTA para cursos de educación continua (2017).

La encuesta permitió conocer impresiones de los escenarios, metodologías y medios tecnológicos utilizados en los cursos de educación continua enfocados en la formación universitaria de las tres modalidades:

- Presencial.

- Híbrida o mixta.

- Virtual. 
Al consultar sobre las modalidades de preferencia para participar en un curso de educación continua, el $84 \%$ de los encuestados manifestó que la modalidad híbrida o b-learning promueve una mayor confianza, ya que en los encuentros o encuadres permiten solventar necesidades y retroalimentar experiencias.

En lo referente a los medios tecnológicos, el 79 \% de los encuestados se sienten cómodos con el uso de plataformas educativas abiertas o de libre acceso (MOODLE 3.2.6); ya que cuenta con herramientas como BigBlueButton (grabación y reproducción de sesiones) para utilización en videoconferencias. Otros complementos son las insignias que permiten hacer un seguimiento y reconocer el esfuerzo (logros) de los participantes; la libreta de calificaciones refleja la puntuación de las actividades realizadas.

Las herramientas y medios utilizados en el aula virtual de la plataforma educativa que consolidaron y sobresalieron en los aprendizajes según los encuestados son:

- Video conferencias $45 \%$.

- Blogs 23\%.

- Foros 10\%.

- Chat $2 \%$.

La Figura 6, refleja a la video conferencia como la herramienta tecnológica de mayor uso en la plataforma educativa, esto se debe principalmente a que los videos pueden ser reproducidos cuantas veces quiera el participante y así consolidar su aprendizaje. 


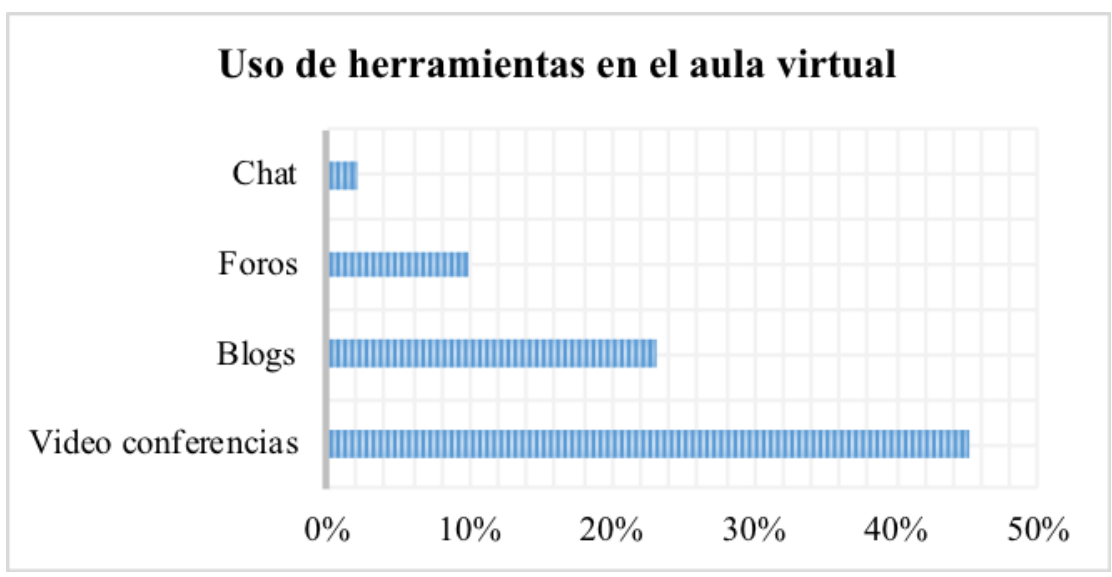

Figura 6. Herramientas y medios utilizados en la plataforma virtual.

Fuente: Sistema informático DEaDV-UTA - Encuesta (2017).

Los participantes encuestados destacaron como elemento diferenciador y que fortaleció el proceso de los cursos de educación continua son la flexibilidad porque cubrió necesidades de los participantes al curso, ritmo propio porque el participante podía disponer del tiempo suficiente para cumplir con las actividades programadas.

\section{CONCLUSIONES}

La Universidad, ante este nuevo escenario y desafío que traen los avances tecnológicos, debe fomentar valores humanísticos que concienticen el buen uso de las tecnologías, así como un pensamiento crítico propositivo que conlleve a una toma de decisiones que fomente soluciones a necesidades y problemas que se presentan en la sociedad.

El docente debe ser flexible a los cambios tecnológicos y evolucionar con los mismos en su rol, pero para llegar a este modelo de enseñanza, las instituciones de educación deben entrenar, motivar y comprometer al catedrático en este nuevo reto.

La relación de la gestión universitaria y tecnológica debe ser de equilibrio como factor clave. Esta debe adaptarse y optimizarse ante el crecimiento de nuevos escenarios y procesos de formación de sus educandos. 
Es primordial validar documentos que permitan obtener las mejores impresiones de los participantes a los cursos de educación continua. Esto sin duda fortalecerá el proceso así como el ambiente del participante para adquirir nuevos conocimientos y competencias para su desarrollo laboral.

Finalmente, la educación 4.0 debe comprometer y asegurar que los aprendizajes tengan factores de flexibilidad, ritmo propio y análisis de datos, lógicamente vinculada a la transformación digital compuesta por tecnología digital, velocidad en los procesos e innovación ante los nuevos desafíos académicos.

\section{REFERENCIAS BIBLIOGRAFICAS}

Bedoya Rodríguez, R. F., et al. (2016). Entornos digitales y políticas educativas : dilemas y certezas. (M. T. Lugo, Ed.) Buenos Aires, Argentina: Instituto Internacional de Planeamiento de la Educación IIPE-Unesco. Recuperado de: http://unesdoc.unesco.org/images/0024/002458/245810s.pdf

Criptonoticias. (2015). criptonoticias.com. Recuperado de: https:/www.criptonoticias.com/ informacion/que-es-una-cadena-de-bloques-block-chain/\#axzz50z9GMUh7

Diaz, L. C. (2014). Investigación en Progreso: Gestión de la Educación Superior en Contextos de Masividad Basada en Tecnologías Inteligentes de Transformación de Información. Latinoamericana de Ingeniería de Software, 2(4), pp. 269-272. Recuperado de: http://revistas.unla.edu.ar/software/ article/view/186

Fierro Santillán, C., y Alfredo Díaz, S. (31 de Julio de 2017). Organización de Estados Iberoamericanos. Recuperado el 4 de Diciembre de 2017, de: http:/www.oei.es/historico/divulgacioncientifica/?Lacuarta-revolucion-industrial-en-la-educacion

Flores Rivera, L. D., y Meléndez Tamayo, C. F. (2017). Variación de la autonomía en el aprendizaje en función de la gestión del conocimiento para disminuir en los alumnos los efectos del aislamiento. RED. Revista de Educación a Distancia, pp. 1-15. doi: http://dx.doi.org/10.6018/red/54/7

Fundación Mapfre. (2017). Libro del profesor El desafio de las tecnologías educación 4.0. Madrid, España: fundacionmapfre.org. Recuperado el 13 de Diciembre de 2017, de: https://www. fundacionmapfre.org/fundacion/es_es/images/libro-profesor-tecnologia_tcm1069-421445.pdf 
Garbanzo-Vargas, G. M. (Enero-Junio de 2016). Desarrollo organizacional y los procesos de cambio en las instituciones educativas, un reto de la gestión de la educación. Educación, 40(1), pp. 67-87. Recuperado el 7 de Diciembre de 2017, de: http://www.redalyc.org/articulo.oa?id=44043204005

García Barbosa, J., García Esteban, R., de Rojas, F. H., López López, V., y Nuñez, M. (2016). Big data. El poder de convertir datos en decisiones. Madrid, España: Telefonica. Recuperado de: https://aunclicdelastic.blogthinkbig.com/ebook-big-data-poder-convertir-datos-decisiones/

Johnson, L. A. (2016). The NMC Horizon Report: Edición Educación Superior 2016. Austin, EE.UU.: The New Media Consortium. Recuperado el 10 de Diciembre de 2017, de: http://cdn.nmc. org/media/2016-nmc-horizon-report-HE-ES.pdf

López Tórrez, Á. y López Herández, L. (2015). Uso de la Educación Virtual en los postgrados de la Facultad de Humanidades y Ciencias Jurídicas de la UNAN-Managua. Universidad y Ciencia, VIII(13), pp. 80-88. Recuperado el 1 de Diciembre de 2017, de: https://revistauniversidadyciencia. unan.edu.ni/index.php/ruc/article/view/102

Majesco. (2016). www.majesco.com. Recuperado de: https:/www.majesco.com/resources/futuretrends-seismic-shift-underway/

Meléndez Tamayo, C., Benítez Aldas, M., Luzuriaga Jaramillo, A., y Flores Rivera, L. (2017). Herramientas sociales como apoyo al profesorado. En: Actas de las Jornadas Virtuales de Colaboración y Formación Virtual USATIC 2017, Ubicuo y Social: Aprendizaje con TIC. (p. 148). Madrid, España: Budok Publishing S.L.

Pérez Juste, R. (1994). Investigación y mejora de programas. Programas comunes y programas personales. En V. García Hoz, Problemas y Métodos de investigación en educación personalizada (pp. 510-536). Madrid, España: RIALP S.A.

Ranz, R. (30 de Mayo de 2016). Una educación 4.0 para el fomento del talento 4.0. Recuperado el 11 de Diciembre de 2017, de: https://robertoranz.com/2016/05/30/una-educacion-4-0-para-el-fomentodel-talento-4-0/ 
Ranz, Roberto. (6 de Junio de 2016). La revolución digital: el impacto de la industria 4.0 en el empleo y la educación. Recuperado de: https://robertoranz.com/2016/06/06/la-revolucion-digital-elimpacto-de-la-industria-4-0-en-el-empleo-y-la-educacion/

Red Universitaria de Educación Continua. (20 de Diciembre de 2017). Definición de educación continua. Recuperado de: http://www.rededucacioncontinua.cl/definicion-de-educacion-continua/

Restrepo,J. M. (12 de Noviembre de 2016). Cuarta revolución industrial y educación. El espectador. Recuperado el 7 de Diciembre de 2017 de: https:/www.elespectador.com/opinion/opinion/cuartarevolucion-industrial-y-educacion-columna-665154

The Flipped Classroom. (2014). Big data ¿Cómo funciona este proceso? Ilustración. Recuperado de: https://i.pinimg.com/736x/11/ef/cc/11 efcc35c42d72b03c77b051f3d55087-big-data.jpg

TYTON Partners. (Enero de 2015). Learning to adapt: a case for accelerating adaptive learning in higher education. Boston, EE.UU.: tyton partners. Recuperado de: http://tytonpartners.com/tytonwp/wp-content/uploads/2015/01/Learning-to-Adapt_Case-for-Accelerating-AL-in-Higher-Ed.pdf

Vivar Zurita, H., Vinader Segura, R., y Abuín Vences, N. (2015). Los MOOCs en la enseñanza superior: una tarea pendiente. Estudios sobre el Mensaje Periodístico, 21(1), pp. 629-642. Recuperado de: http://eprints.ucm.es/33618/1/MOOCs-Natalia\%20Abuin.pdf

Zapata-Ros, M. (2014). Los MOOC en la crisis de la Educación Universitaria. Docencia, diseño y aprendizaje. Murcia, España: C. I. Platform. 